\title{
Design de sistemas para análise do ciclo de vida de um produto: slow fashion
}

\author{
Conti, Flávia ${ }^{a}$ Figueiredo, Luiz Fernando $^{\text {b }}$ \\ ${ }^{\text {a } U n i v e r s i d a d e ~ F e d e r a l ~ d e ~ S a n t a ~ C a t a r i n a, ~ B r a s i l, ~ f l a v i a p e r e i r a c o n t i @ g m a i l . c o m ~}$ \\ ${ }^{\mathrm{b}}$ Universidade Federal de Santa Catarina, Brasil, 1ff@cce.ufsc.br
}

\section{Resumo}

O artigo descreve o processo de reavaliação e esmeração de um sistema de produção da microempresa de semijoias Cantrelle Design, com o objetivo de otimizar a estrutura organizacional e de produção por meio do design de sistemas e o slow fashion. $O$ design de sistemas por considerar o produto como um conjunto inteiro, e o slow fashion, porque visa a democratização do processo de criação de peças de forma mais lenta, preocupando-se com o desenvolvimento dos processos. Para alcançar um resultado satisfatório, utilizou-se a metodologia desenvolvida por Ezio Manzini e Carlo Vezzoli, o Life Cycle Design (LCD), procurando reduzir os inputs e outputs o máximo possivel, tanto em termos quantitativos quanto qualitativos. Ponderando assim, a nocividade de seus efeitos, por meio da avaliação de todas as fases do produto, que são subdivididas em pré-produção, produção, distribuição, uso e descarte. Por se tratar de semijoias, sendo, então, um bem durável, requereram-se poucos recursos durante o uso e manutenção, concentra-se em reduzir o impacto nas fases antecedentes e posteriores ao uso. Como resultado, obteve-se uma potencialização na gestão da empresa, reduzindo os gastos energéticos e materiais. Atingiu-se tal solução por meio de uma melhor organização de etapas operacionais nas fases antecedentes ao uso do produto, buscando adequar-se ao sistema slow fashion, com a otimização do volume de compras e logística de vendas, reavaliação da embalagem e material aplicados. Percebeu-se que a matéria prima já em uso é a menos impactante para o ambiente por ser de alta durabilidade e passivel de reaproveitamento. Por fim, redesenhou-se a embalagem com tecido reciclado, de uma forma que possa ser reutilizada pelo consumidor final após ser adquirida. Conclui-se que é possivel readequar um sistema já em andamento, adaptando-o de forma a reduzir seu impacto na natureza por meio do slow fashion e design de sistemas, valorizando o processo de produção, não só o lucro financeiro que a venda do produto proporciona, além de aperfeiçoar o sistema como uma unidade e repensar o conjunto para 
valorizar a qualidade e o modo de produção, expondo a possibilidade de renovar o sistema industrial vigente de modo sustentável e consciente, por meio de uma ação local, visando atingir um macro sistema de forma harmônica.

Palavras-chave: slow fashion, design de sistemas, semijoias, sustentabilidade, metodologia.

\section{Abstract}

The article describes the process of reevaluation and refinement of a production system of the semi-jewelry company Cantrelle Design, with the objective of optimizing the organizational and production structure through system design and slow fashion. The design of systems considering the product as a whole, and slow fashion, because it aims to democratize the process of creating parts more slowly, worrying about the development of processes. To achieve a satisfactory result, the methodology developed by Ezio Manzini and Carlo Vezzoli, Life Cycle Design (LCD), was used to reduce inputs and outputs as much as possible, both in quantitative and qualitative terms. Thus, the harmfulness of its effects is evaluated by means of the evaluation of all phases of the product, which are subdivided into preproduction, production, distribution, use and disposal. Because it is a semijewelery, being a durable good, few resources were required during use and maintenance, it focuses on reducing the impact in the antecedent and postuse phases. As a result, the management of the company was strengthened, reducing energy and material expenses. This solution was achieved through a better organization of operational stages in the phases prior to the use of the product, seeking to adapt to the slow fashion system, with the optimization of the volume of purchases and sales logistics, revaluation of the packaging and material applied. It was noticed that the raw material already in use is less impacting to the environment because of it's high durability and reusable. Finally, the packaging has been redesigned with recycled fabric in a way that can be reused by the final consumer after being acquired. It is concluded that it is possible to readjust a system already in progress, adapting it in order to reduce its impact in nature through the slow fashion and systems design, valuing the production process, not only the financial profit that the sale of the product provides, in addition to improving the system as a unit and rethinking the whole to value the quality and the way of production, exposing the possibility of renewing the current industrial system 
in a sustainable and conscious way, through a local action, aiming to achieve a macro system.

Keywords: slow fashion, systems design, semijoias, sustainability, methodology.

\section{Introdução}

Atualmente, consomem-se $30 \%$ mais recursos naturais do que a capacidade de renovação da Terra e, caso continuemos nesse ritmo, será imprescindível que tenhamos um segundo planeta igual ao nosso para atender nossas necessidades de matéria-prima, alimento e energia (Ministério do Meio Ambiente do Brasil, 2017). Para tanto, faz-se fundamental uma mudança no nosso sistema de produção e consumo, necessitando-se de uma reavaliação sobre o assunto em salas de aula, ambientes familiares, empresas e governos.

Manzini e Vezzoli (2005) apontam que a sustentabilidade é um objetivo a ser alcançado, não somente uma direção a ser seguida, como se entende hoje. Os autores apontam requisitos a serem atendidos para dizer-se sustentável, valendo ressaltar tais como otimizar o emprego de recursos não renováveis (água, terra e ar), não acumular lixo que o ecossistema não seja capaz de renaturalizar (fazer retornar às condições originais) e faz uso de recursos renováveis, garantindo a renovação.

Tratando-se de empresas, a revista Exame (2016) apontou entre as 100 empresas mais sustentáveis do mundo nomes como BMW (automóveis), Adidas (têxtil e vestuário) e Coca-Cola Enterprises (bebidas), tendo em comum a tomada de medidas preventivas, com performances notáveis nas áreas de energia, resíduos e redução no uso de água. Constata-se a preocupação destas empresas em empenhar seus esforços na reavaliação do sistema de produção como um todo, não somente na recuperação de danos já causados - soluções endof-pipe.

No slow fashion, encontra-se diversas semelhança com o que Manzini e Vezzoli ressaltam como de suma importância ao aplicar o design de sistemas em uma empresa, destacando, como principal delas, a preocupação em pensar o sistema de produção como um conjunto completo, possuindo impactos em todas suas etapas (DELLA MEA, 2012). Por tanto, torna-se relevante o uso do movimento slow fashion para um negócio de moda, trazendo um aperfeiçoamento técnico e, consequentemente, uma redução de custos.

Este trabalho tem como fim exemplificar a aplicação do slow fashion no ateliê de semijoias Cantrelle Design e o resultado de tal trabalho, partindo de uma pesquisa bibliográfica 
inicial e aplicação de uma metodologia de design de sistemas com enfoque na sustentabilidade.

\section{Procedimentos Metodológicos}

Este estudo pode ser classificado como de natureza descritiva, abordagem qualitativa (contém análise não generalizáveis pela estatística), e com objetivos descritivo e explicativo, buscando uma nova visão sobre o design de sistemas, com enfoque no slow fashion, tornando-se viável a aplicação desta associação para o mundo empresarial. Para tanto, utilizou-se de uma pesquisa bibliográfica e estudo analítico (Lakatos \& Marconi, 2003), atentando-se a problemática do sistema de produção de moda atual e como o design de sistemas voltado para o slow fashion pode contribuir de modo positivo para o meio socioambiental, trabalhando a problemática de como se pode modificar o sistema de produção de uma empresa tornando-a mais sustentável e eficaz?

Considerando os fatores que se deseja atender com a pesquisa, utilizou-se a metodologia desenvolvida por Manzini e Vezzoli (2005), o Life Cycle Design (LCD), que objetiva a redução de carga ambiental vinculada a todo o ciclo de vida de um produto, desde a compra de matéria prima, até seu descarte. Para uma melhor visualização de toda a metodologia, a figura 1 ilustra a abordagem e objetivo do LCD e a figura 2 demostra suas estratégias durante seu processo.
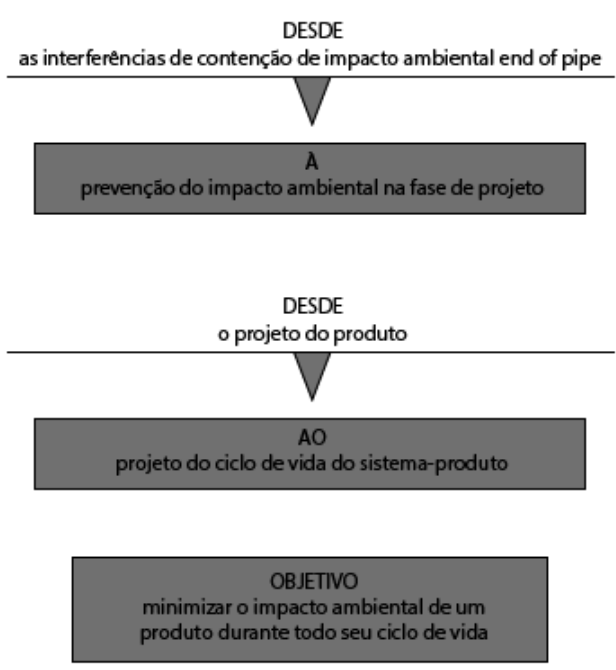

Fig. 1 Abordagem e objetivos LCD 


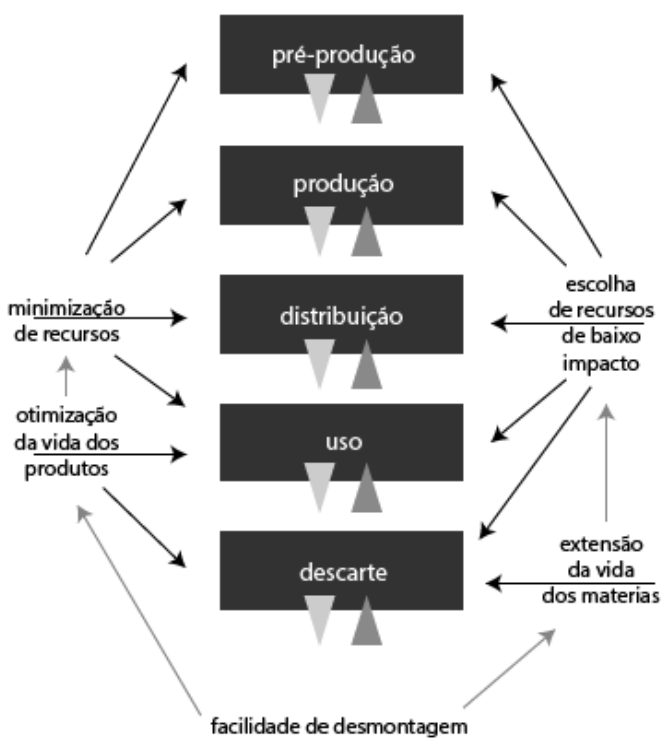

Fig. 2 Estratégias LCD

\section{Design de Sistemas Para Sustentabilidade}

Existindo desde o princípio da humanidade, os sistemas são estudados para compreender e aprimorar a complexidade dos seres vivos, da natureza e da ciência em si. No âmbito do Design, existe uma geração inevitável de novos planos latentes no processo de design, incitando novas estratégias de gestão em cada etapa do processo para se obter melhores resultados no produto final (Bastagnino, sem ano).

Desde o surgimento dos debates sobre sustentabilidade, o design vem ganhando papel de cada ver maior importância e responsabilidade, demandando-se a aplicação de soluções sustentáveis no desenvolvimento de projeto, desde sua matéria prima até seu descarte (Della Mea, 2012). Para tal, Vezzoli (2010) aponta que o designer tem de considerar o produto como um sistema, integrado de diversas partes, abordagem conhecida também como Sistema Produto-Serviço (PSS), onde se considera não somente o produto final em si, mas tudo o que ele demanda de gasto de energia, matéria-prima, ferramentas de produção (descartáveis ou não) e subprodutos para sua funcionalidade. O PSS trabalha em níveis mais estratégicos, com produtos-serviços intrinsicamente sustentáveis e desmaterialização do consumo. 
Tal discussão ainda é recente, demandando mudanças socioculturais por parte dos consumidores para haver resultados mais assertivos, fazendo-se necessária a busca constante por novas formas de produção, tecnologias e conectividade com outras empresas, criando uma união em busca de uma sociedade mais correta e sustentável.

\section{Slow Fashion}

Em 2002, Kakegawa, uma cidade japonesa decidiu por abolir o máximo possível do uso de transportes, construir casas de bambu e buscar negociar as horas de trabalho, tornando-se, então, a primeira cidade a se denominar slow no mundo. Que isso tenha acontecido no país mais workaholic do mundo, foi surpreendente, mas mais do que isso, fez muitos ao redor do mundo questionarem sobre seu estilo de vida e o que deve-se priorizar no cotidiano (Sarate, 2011).

Vivemos em um período em que estar sempre ocupado é sinônimo de sucesso, mesmo que não se sabe exatamente o porquê ou a finalidade de tudo isso. Bauman (2001) já apontou que é necessário que o ser humano descanse o corpo e a mente para poder desfrutar de suas tarefas, achar um ponto de equilíbrio entre o ócio e o trabalho, apreciando cada momento.

Vê-se, então, pessoas e grupos já em busca de novos caminhos para se obter uma vida mais equilibrada, perseguindo uma maior qualidade para sua vida e seu tempo. O slow life, tendo suas primeiras aparições nos anos 80 na Itália, ganha força no século XXI e é uma corrente que desafia o excesso em que se vive na cultura contemporânea, tendo como acrônimo para "sustentável, local, orgânico e whole" (BUENO, 2016). Vem tendo grande força em áreas como moda, educação, alimentação, entre outros.

O slow fashion é a busca no fortalecimento da conexão entre o consumidor do produto e o designer criador, também diligenciando modos de redefinir os valores sociais em relação ao consumo de moda. Sendo assim um movimento que prioriza o esmero, a qualidade e a produção pensada a longo prazo (JOHANSSON apud. SILVA et al., 2016).

O designer responsável busca pensar todo o processo de produção, desde de suas matérias primas até o descarte do produto, explorando opções que conscientize o cliente sobre o novo modelo de agir em relação ao consumo (SILVA et al., 2016). Concentra-se em criar produtos com design atemporal, de qualidade e evidenciando o aspecto emocional, criandose vínculo com o produto seja por meio do seu material, modelagem ou conforto (Della Mea, 2014).

Nota-se uma preocupação com o meio-ambiente e com a saúde mental do ser humano quando se trata do slow life, tendo o slow fashion como aplicação, pensando no conforto em que usufruir-se-á e em seu impacto ambiental, empenhando-se em criar um produto com material sustentável e durável. 


\section{Análise de Fatores}

Baseando-se, então, na metodologia de Vezzoli e Manzini (2005), o Life Cycle Design (LCD), fez-se uma avaliação de cada etapa de produção dos produtos confeccionados pelo ateliê de semijoias de Cantrelle Design, com sede em Florianópolis. Por se tratar de semijoias, sendo, portanto, um bem durável, requereram-se poucos recursos durante o uso e manutenção, concentra-se em reduzir o impacto nas fases antecedentes e posteriores ao uso, sendo elas as fases de pré-produção, produção, distribuição e descarte, sendo essas as etapas que serão tratadas no percurso da análise.

Vezzoli e Manzini (2005) apontam em seu livro que é muito improvável que, ao analisar os fatores da produção, será adotada apenas uma estratégia para satisfazer todos os requisitos ambientais, uma vez que o uso de diversas destas podem se tornar sinergéticas, mas sempre em mente os objetivos que elas irão cumprir, com o risco de tornar-se desvantajoso para a empresa em caso de se escolher incorretamente as estratégias à serem utilizadas.

Para precaver tais erros de escolha e aplicação de estratégias, foram utilizadas algumas metodologias e instrumentos de análise e suporte às decisões de projeto, como o Life Cycle Assessement (LCA).

\subsection{Pré-produção}

A primeira etapa diz respeito a fase em que são produzidos os materiais, ou seja, a matéria prima que será utilizada na produção. O material já em uso é o latão, um metal com liga de cobre com zinco, de recurso primário não renovável, por ser extraído do solo, sendo de alto impacto de acordo com método Ecoindicador 95 (Manzini e Vezzoli, 2005), porém de alta durabilidade, compensando o impacto no seu descarte. Tal material é considerado de recurso primário - ou virgem - e pode vir a se tornar recurso secundário - ou reciclado com a aplicação de uma ação que recolha o material que seria descartado - pós consumo e venha a ser reutilizado.

Utiliza-se também cristais e pedras sintéticas para adorno, considerados recursos primários renováveis por serem fabricados em laboratório, sendo estas últimas de material reciclado, pois sua fabricação deriva do pó de pedras naturais que são pulverizadas e reconstruídas, tornando-as de baixo impacto e de alta durabilidade.

\subsection{Produção}

No processo de produção, consideram-se três momentos, sendo eles a transformação dos materiais, a montagem e o acabamento. Adicionam-se aqui as atividades de pesquisa, desenvolvimento e projeto.

A pesquisa é realizada de duas formas: online e off-line. Na fase online pesquisa imagens e comportamentos à nível global que possam ser afins ao público alvo e na fase off-line se 
vai às ruas pesquisa registrar por meio de fotos, vídeos ou desenhos tendências locais. Após a coleta de dados desenvolve-se um painel visual que irá guiar o desenvolvimento. Nesta etapa nota-se um gasto energético razoável, por se utilizar de ferramentas eletrônicas para efetuar o trabalho. Não se tem ainda outras ferramentas que possam substituir as já utilizadas atualmente com o intuito de reduzir os gastos energéticos, cabendo somente à indústria a criação de novas tecnologias que possam cumprir tal função.

As fases de desenvolvimento e projeto são feitas por meio da técnica de brainstorming, criando-se o maior número possível de alternativas de produto, fazendo-se a escolha das melhores alternativas criadas e, por último, o desenho refinado destas. Utiliza-se como materiais o papel, lápis e lápis de cor. O papel é destinado posteriormente para a reciclagem, mas pode-se diminuir o uso deste por meio da digitalização ao fazer o desenho refinado em programas de design específicos pra tal função.

O material já é adquirido trabalhado - chapa, fio, tubo, etc. - para reduzir o tempo de trabalho, o que também implica na não reutilização de escopo. Para reduzir tal inconveniente, guarda-se o excesso para a criação de peças únicas posteriormente, reduzindo o descarte em mais da metade do que seria rejeitado.

A montagem do produto se realiza manualmente, tendo como fonte de energia para trabalhar o material o fogo, fazendo uso de nenhuma energia artificial, logo, foca-se nesta fase em reduzir o material utilizado, as perdas e refugos, desenvolvendo-se peças minimalistas dando ênfase ao modo de produção e qualidade do produto final.

O acabamento a única etapa realizada com uma ferramenta elétrica, um aparelho de mandril, sendo que este realiza tarefa intransferível, uma vez que desempenhar a mesma função à mão seria mais trabalhoso e demorado, influenciando diretamente no preço final, o que não é pretendido.

Por fim, o produto é enviado para um serviço terceirizado para aplicar o chamado banho de ouro, onde o produto é revestido por uma camada fina de ouro 18 quilates. O processo é realizado por um parceiro da região, evitando gastos energéticos com deslocamento. Faz-se o envio de uma quantidade razoável de peças por remessa, o que diminui também os gastos de material e energia durante o processo, impactando diretamente no preço e nos impactos ambientais.

\subsection{Distribuição}

Esta fase foi leva em consideração a embalagem, o transporte e armazenagem, sendo esta que mais demonstrou dificuldade em ser trabalhada, pois o ateliê trabalha com venda online e atendimento a domicílio, exigindo duas embalagens distintas, uma para presente e outra para envio no correio. 
A embalagem anterior utilizada era composta por sacola de papel, com etiqueta e fita de tecido, contendo no interior papel seda para proteger o produto e também servir como fator estético, sendo adicionado um pequeno informante, também de papel, com dicas de como cuidar da peça e a data de garantia desta.

Preocupando-se em reduzir a quantidade de material utilizada e também em reduzir o valor gasto com cada embalagem, desenvolveu-se uma em papel vegetal, feita à mão e fechada com um selo adesivo, obtendo-se uma redução considerável de material e uma queda de $83 \%$ do valor gasto com a embalagem. A figura 3 ilustra como era e como ficou a embalagem para presente.
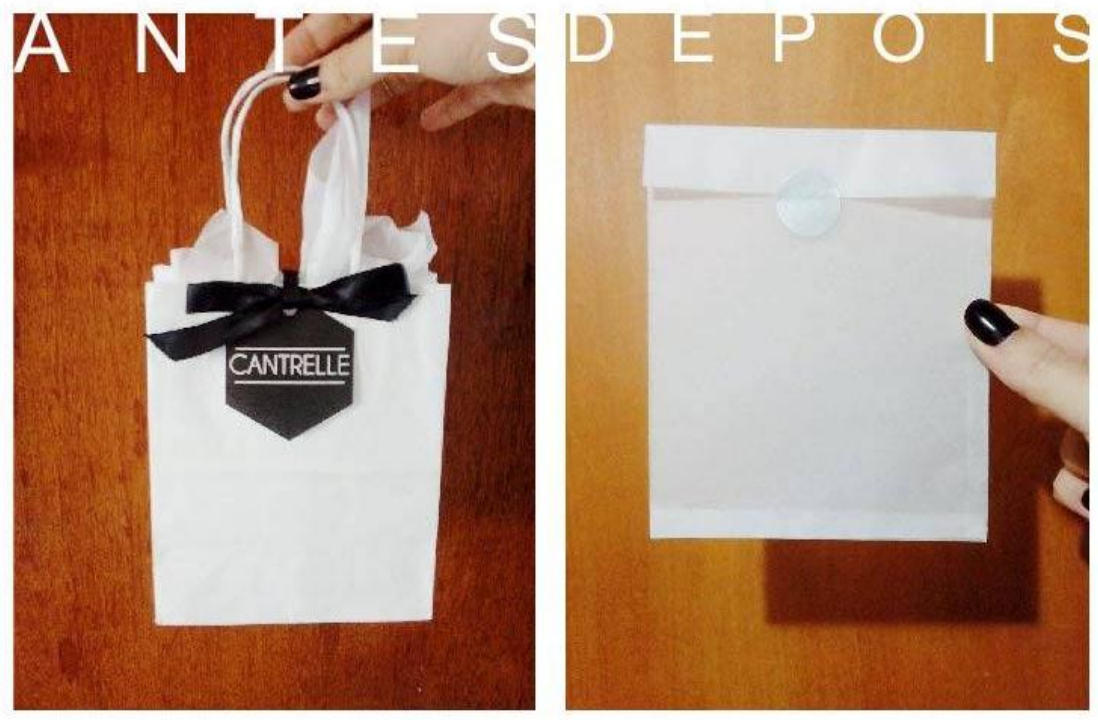

Fig. 3 Embalagem presente

A embalagem do correio é padronizada pela própria agência do Correios, não podendo haver modificações no seu exterior - utiliza-se uma caixa de papelão com medidas de 20x11x7,5 cm. Porém, no interior, utilizava-se papel seda, um pequeno embrulho de tule (tecido vazado) e finalizado com fita de tecido para finalizar. A mudança foi pouca, retirando somente o embrulho de tule, que era o com maior impacto ambiental, e reduziu-se a quantidade que se utilizava do papel seda pela metade e, como consequência a quantidade de fita. Pode-se notar a mudança na figura 4. 

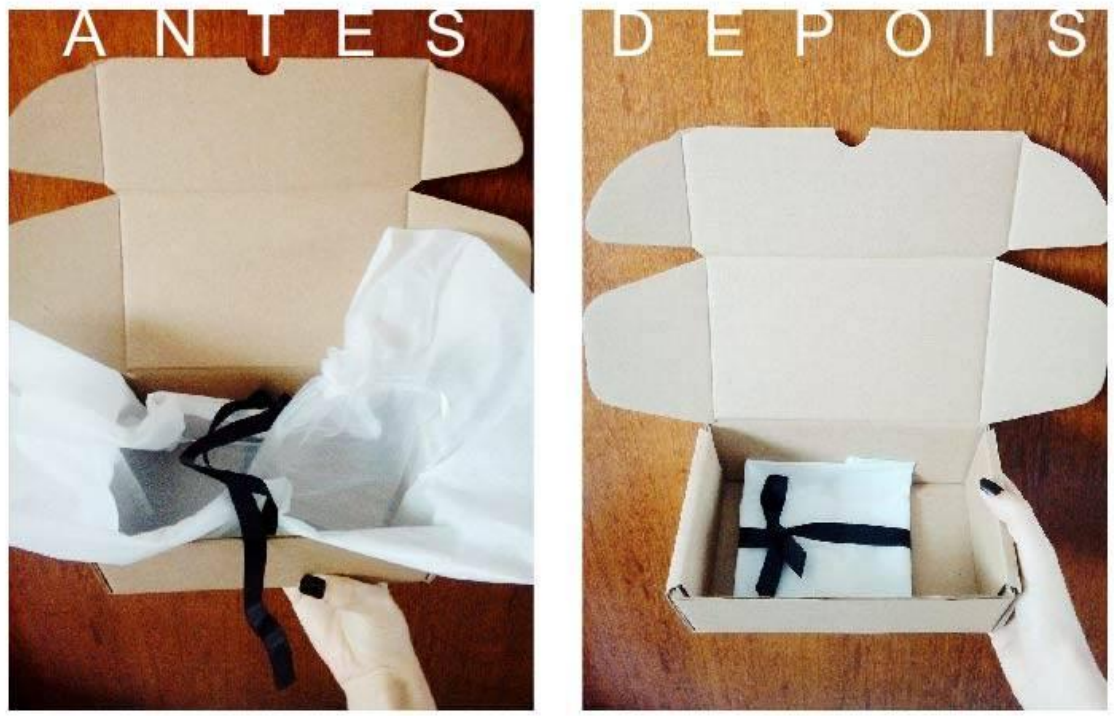

Fig. 4 Embalagem correio

Como meio de distribuição, utiliza-se entrega em mãos nas cidades de Florianópolis e Criciúma, evitando o uso de transportes poluentes e, para o resto do Brasil, ainda limita-se a opção do Correios, única empresa que abarca todas as cidades do país em um prazo razoável.

Por fim, a armazenagem do produto, por ser de pequeno porte, é conservado no próprio ateliê, em mostruários de tecido que são utilizados para também levar para as casas dos clientes que optam pelo atendimento à domicílio.

\subsection{Descarte}

Esta fase é, para Manzini e Vezzoli (2005), a que merece maior atenção por ser ela com maior possibilidade de impacto ambiental e por envolver tanto quem projeta quanto quem produz. Como citado anteriormente, buscou-se algumas soluções para prolongar o descarte das peças, levando em consideração três fases: a fase imediata, que diz respeito aos produtos produzidos anteriormente às mudanças no sistema de produção e estão sendo descartados atualmente; a fase de curto período, condizente aos produtos que estão sendo projetados atualmente e serão descartados em breve; e a fase de médio/longo prazo, relacionada a produtos em que atualmente se pode iniciar uma concepção mais profunda, prolongando a vida útil.

As soluções geradas foram o serviço assistência técnica vitalícia (fases imediata e de curto prazo), onde, em caso de danos, o cliente pode solicitar o reparo da peça pelo preço de 
custo, e o retorno da peça caso não se deseje mais (fases de curto e médio prazo), com o intuito de poder reutilizar o material (reciclagem em anel fechado), sendo neste caso fornecido um desconto para o consumidor que efetuar a devolução.

A empresa espera que, com tais ações, haja um maior vínculo emocional com a marca, maior conscientização por parte de quem compra e um aumento em até 5 anos de uso das peças - tempo que varia de acordo com o cuidado do usuário.

\section{Resultados}

Como resultado, obteve-se uma potencialização na gestão da empresa, reduzindo os gastos energéticos e materiais, com números significativos como queda de $83 \%$ no valor gasto com as embalagens, uma porcentagem de $38 \%$ das encomendas sendo reparos de peças nos últimos três meses, não só de produção própria, como também de outras empresas, $23 \%$ das compras provenientes de indicações de outros clientes e uma otimização da produção em $16 \%$.

Também é relevante citar que a empresa tem buscado levar mais informações sobre o processo de produção para o cliente, fazendo este criar vínculos emocionais com a marca e instruindo-o sobre a importância do pensar consciente e da seriedade por trás da criação e desenvolvimento de cada produto, obtendo feedbacks qualitativos de significativa importância, como uma relação mais aberta com o cliente e nenhuma reclamação feita nos últimos 6 meses desde que aplicou-se as estratégias.

\section{Conclusão}

Conclui-se, portanto, que ao aplicar o modelo slow fashion de produção por meio da metodologia LCD pode-se produzir um produto de apelo estético e também pensando nos impactos socioambientais, não só com foco na peça final, mas em toda a cadeia de produção, fazendo uma análise do sistema e adotando estratégias em cada fase da fabricação que, em conjunto, reduzem o uso indevido e em excesso de material e energia, minimizando, consequentemente, os gastos financeiros.

O presente artigo salienta a urgência de revisão do sistema de consumo atualmente em vigor, pondo em pauta a necessidade não só das empresas de reavaliarem o modo em que operam como também do consumidor buscar adquirir produtos de marcas que se preocupam com causas ambientais, carecendo, como ditas por Manzini e Vezzoli (2005), que o indivíduo e a sociedade possuam feedbacks ambientais corretos, que sejam oferecidas alternativas sistêmicas socialmente aceitáveis e favoráveis ao ambiente e que "se desenvolva uma cultura adequada para interpretar corretamente os feedbacks ambientais e 
reconhecer o valor das alternativas propostas". E para que tudo isso ocorra, faz-se imprescindível o envolvimento político, industrial e social, por meio de práticas diárias, podendo, assim, encurtar o caminho entre uma sociedade consumista para uma sociedade que coexiste com todos os seres vivos, vendo-os como iguais e não mais como matéria prima para saciar seus desejos e impulsos.

\section{Referências}

Barbosa, V. (2016). As 100 empresas mais sustentáveis do mundo em 2016. Disponível em http://exame.abril.com.br/negocios/as-100-empresas-mais-sustentaveis-do-mundo-em-2016

Bastagnino, L. (2016). Design di sistemi i sistemi industriali aperti: um nuovo approccio al progreetto, un nuovo modello di business. 26 slides. Apresentação em Power Point.

Bauman, Z. (2001). Journal of consumer culture. Home: Geocities. Disponível em http://www.geocities.ws/visisto/Biblioteca/BAUMANN_Consuming_Life.pdf

Bueno, B. (2016). Movimento slow life: desacelerando a vida. Disponível em https://pt.linkedin.com/pulse/movimento-slow-life-desacelerando-vida-b\%C3\%A1rbaramantovani-bueno

Della Mea, L. (2012). Design de sistemas para a sustentabilidade. Disponível em http://www.autossustentavel.com/2012/06/design-de-sistemas-para.html

Della Mea, L. (2014) A moda em [re]evolução: slow fashion. Disponível em http://www.autossustentavel.com/2014/05/a-moda-em-revolucao-slow-fashion.html

Manzini, E.; Vezzoli, C. (2005). O desenvolvimento de produtos sustentáveis: os requisites ambientais dos produtos industriais. São Paulo. Editora da Universidade de São Paulo.

Marconi, M.; Lakatos, E. (2003). Fundamentos de metodologia científica. $5^{\text {a }}$ edição. São Paulo. Atlas S.A. O que é consume consciente? (2017). Disponível em http://www.mma.gov.br/informma/item/7591-o-que-\%C3\%A9-consumo-consciente

Papanek, V. (1985). Design do the real world: human ecology and social change. Londres. Thames \& Hudson Ltd. O conceito de fast fashion (2010). Disponível em https://www.revide.com.br/editorias/moda/o-conceito-de-fast-fashion/

Sarate, F. (2009). O movimento slow life e a desaceleração da sociedade de consume contemporânea.
Disponível
em
$\mathrm{http}: / /$ www.comunicacaoetendencias.com.br/wp- content/uploads/2011/04/TCC-Fernanda-Sarate.pdf

Silva, S.; Busarello, R. (2016). Fast fashion e slow fashion: o processo criativo na contempotaneidade.

Disponível

em

http://www.usp.br/estetica/index.php/estetica/article/view/48/38

Vezzoli, C. (2010). Design de sistemas para a sustentabilidade: teoria, métodos e ferramentas para o design sustentável de "sistemas de satisfação". Salvador. EDUFBA. 